\title{
Lattice Boltzmann modeling and simulation of isothermal drying of capillary porous media
}

\author{
Zachariah, G. T..$^{\text {; }}$ Panda, D. ${ }^{\text {a }}$ S Surasani, V. J. ${ }^{a^{*}}$
}

a Department of Chemical Engineering, Birla Institute of Technology and Science, PilaniHyderabad Campus, Hyderabad, India, 500078

*E-mail of the corresponding author: surasani@hyderabad.bits-pilani.ac.in

\begin{abstract}
Modeling of dring of capillary porous media is difficult due to the complex and coupled heat and mass transfer that occur at dynamic liquid-gas-sold interface. Thus far, drying was simulated using either continuum models or pore-network models, both of which have limitations. In this work, the Lattice Boltzmann Method (LBM) is used to simulate the drying in porous media. The LBM is ideal for such simulations as it can incorporate complex effects in a simple way to exhibit realistic fluid-gas interface during drying of capillary porous media.
\end{abstract}

Keywords: Lattice Boltzmann Method; Capillary Porous media; Drying, Pore Network. 


\section{Introduction}

Drying of porous media is one of the most energy intensive operations in numerous industries such as textile, food, agriculture and etc. Experimental methods have been established to understand the macroscopic drying kinetics, whereas experimentation for the detailed micro scale transport behavior such as phase and temperature distributions and the corresponding micro-macro interactions still need significant scientific development. Modeling and simulation provides an efficient method for investigating such microscopic behavior. Simulation of the drying of porous media is a challenging task as it involves mass, momentum and heat transfer in a three-phase system (solid, liquid and gas) [1]. Various modeling techniques have been used in the past to simulate such complex processes including classical continuum methods [2], discrete Pore Network Models (PNM) [1] and more recently, the Lattice Boltzmann Method (LBM) [3]. Classical continuum models simulate the complex behavior of the fluid in the porous media macroscopically. These models are derived from either homogenization [2] or volume averaging techniques [4]. The continuum assumption in these models imposes an additional length scale constraint on its application. Moreover, models based on Darcy's law ignore nonequilibrium and inertial effects as the flow is at low Reynolds numbers.

A more promising alternative is the discrete pore network model, which is derived from statistical physics concepts like percolation theory, fractal concepts and scaling theory[1]. These involve representing the void space in a porous medium as a pore network and solving the governing equations on this network. Discrete pore network models are especially useful when the effects of pore space or long-range correlations are strong. Despite its various advantages, the pore network models have numerous shortcomings that need to be addressed. For one, the actual porous media has to be converted into a pore network before simulating which is a complex and time-consuming process, especially for more complicated and broad pore size distribution. Further, such models have always assumed either the pore or the throats to be of zero volume which is unrealistic [1]. The fluid-air interface develops dynamically with time and depends on the capillary pressures and the pore and throat geometry. The inclusion of these effects is necessary to improve the accuracy of the pore network model. The Lattice Boltzmann Method provides an alternative by accommodating the actual geometry of porous media.

The LBM is a relatively new mesoscopic method [3] which has repeatedly proven its ability to simulate transport in porous media. It involves solving the discrete Boltzmann equation to reproduce the Navier-Stokes equation in the continuum limit. This can be shown by conducting a Chapman Enskog expansion of the Boltzmann equation [5]. It is simple and accurate to incorporation of complex geometries. And LBM is efficient with parallelization capabilities which make it ideal for simulations in porous media. Further, due to its roots in 
statistical physics, it can easily simulate multi-physics processes including multiphase and multi-component flow, cavitation etc. without explicitly tracking the interface as is done in continuum and pore-network models.H. El. Abrach, et. al.[6] investigated the drying of deformable porous media using LBM, macroscopically. Sukop et.al. [7] demonstrated the applicability of the Lattice Boltzmann method in simulating several micro-scale porous phenomenon such as adsorption, wetting, liquid retention and capillary condensation. The present work explains on how LBM can simulate the intricate microscopic interactions between such phenomenon, leading to complex fluid behaviour, such as capillary pumping and haines jumps [8], in non-deformable porous media.

\section{Lattice Boltzmann Method (LBM)}

In LBM, the motion of fluid is described by a set of particle distribution functions (PDF), which helps quantify the number of particles with a particular velocity at a certain location in space. The evolution of this PDF with time is described by the lattice Boltzmann equation (LBE) with, in this case, the Bhatnagar-Gross-Krook (BGK) collision operator [9]. The LBE is written as

$$
\frac{\partial f_{k}}{\partial t}+c_{k} \frac{\partial f_{k}}{\partial x_{i}}=\Omega_{k}(f)+F_{k}
$$

Where, $f_{k}$ is the particle distribution function in the kth direction, $c_{k}$ is the velocity in the kth direction, $F_{k}$ is the forcing term and $\Omega_{k}$ is the collision operator given by

$$
\Omega_{k}(f)=-\frac{1}{\tau}\left(f_{k}-f_{k}^{e q}\right)
$$

Where, $\tau$ is the relaxation time and is the equilibrium distribution function given by the Maxwell Boltzmann Distribution, which can be approximated into the following simple form:

$$
f_{k}^{e q}(r, t)=\rho w_{k}\left(1+3 e_{k} u+\frac{9}{2}\left(e_{k} u\right)^{2}-\frac{3}{2} u^{2}\right)
$$

The LBE is solved by discretizing the space, time and the velocity. Various different velocity discretization schemes have been developed. In this study, we use the twodimensional nine velocity model (D2Q9).

For this scheme, the discrete velocities, and are given by: 


$$
e_{k}=\left\{\begin{array}{cl}
(0,0) & k=0 \\
( \pm 1,0) c,(0, \pm 1) c, & k=1,2,3,4 \\
( \pm 1, \pm 1) c, & k=1,2,3,4
\end{array} \quad w_{k}=\left\{\begin{array}{cc}
4 / 9 & k=0 \\
1 / 9, & k=1,2,3,4 \\
1 / 36, & k=1,2,3,4
\end{array}\right.\right.
$$

The macroscopic properties we require, such as the density and velocity are derived from the PDF as

$$
\rho=\sum_{n=1}^{n} f_{k} \quad \rho u=\sum_{n=1}^{n} e_{k} f_{k}
$$

\subsection{Incorporating Phase Change Models}

In LBM, phase separation is achieved by incorporating a force on the particles at a node based on the particle density in the adjacent nodes, i.e., fluid in one node will experience a force in the direction with the higher neighboring density. In this study, the interaction force is incorporated into the model by shifting the velocity in the equilibrium distribution as given below:

$$
u^{e q}=u+\frac{\tau F_{\text {int }}}{\rho(x)}
$$

It is also possible to incorporate the interaction force via direct body forcing, where an additional term is added after the collision process [10]. The most commonly used method is the one proposed by Shan and Chen for Multiphase simulation[11]The inter-particle force is then taken as:

$$
F_{\text {int }}(x)=-G \psi(x) \sum_{k=1} w_{k} \psi_{k}\left(x+e_{k}\right) e_{k}
$$

Where, $\mathrm{G}$ is an interaction strength constant and is either negative or positive for attraction and repulsion respectively, $w_{k}$ is the weight function given in equation 4 and $\psi$ is the effective mass, a function of density. This formulation results in an equation of state given by:

$$
P=c_{s}^{2} \rho+\frac{c_{0}}{2} g[\psi(\rho)]^{2}
$$

Where, cs is the velocity of sound, $c_{0}$ is a parameter determined by the chosen lattice structure and is a parameter that controls the magnitude of the inter-particle forces. 
The formulation of $\psi$ can be designed to incorporate a more accurate Equation of State if required[12]. Equation 8 can be rearranged to give:

$\psi(\rho)=\sqrt{\frac{2\left(p_{E O S}-c_{s}^{2} \rho\right)}{c_{0} g}}$

Here, $\mathrm{P}_{\mathrm{EOS}}$ is the equation of state used, which, in this case, is the Carnahan Starling equation of state, given by:

$$
p_{E O S}=\rho R T \frac{1+b \rho / 2+(b \rho / 4)^{2}-(b \rho / 4)^{3}}{(1-b \rho / 4)^{3}}-a \rho^{2}
$$

Applying to equation 7 and combining the numerical approximations of the gradient [13], we get:

$$
F_{\text {int }}(x)=c_{0} \beta G \nabla \psi(x)-(1-\beta) c_{0} G \Delta \nabla \psi^{2}(x) / 2
$$

Where $G$ is taken as -1 to ensure a positive value under the root and is a weighting factor that can be tuned for each equation of state. For the CS equation of state $\beta$ is taken as 1.16.

Here, wall interaction is incorporated in a similar way, by assuming the walls to have density equal to the liquid density. Therefore, $\rho_{\text {wall }}=\rho_{\text {liquid }}$. This gives the formulation:

$$
F_{a d s}(x)=-G_{a d s} \psi(x) \sum_{k=1}^{n} w_{k} \psi_{\text {wall }}\left(x+e_{k}\right) e_{k}
$$

\section{Simulation Results and Discussion}

In this study, we simulated the drying of two types of porous media: structured and irregular. The structured porous medium consisted of equally spaced horizontal and vertical throats intersecting in circular pores, while the irregular porous media did not exhibit any observable pattern. Figure 1 shows the simulated results obtained using Lattice Boltzmann method. In the porous medium as shown in Figure 1A, the radius of the pores are much higher than that of the throats. This implies that there is a sudden and large increase in radius of the meniscus, thereby making it unstable/instabilities during drying. Therefore, the interface moves rapidly to the next stable orientation. This causes the surrounding liquid to be pumped outward and thus causing smaller pores and throats to be filled. This phenomenon can be observed in Figure 1A. The emptying of the pore circled in red leads to the refilling of the vertical throat circled in blue. Such effects are more prominent and discernable near the edges of the porous media where the pumping is only possible in limited directions. This phenomena of Haines jump events can significantly change the liquid orientation in the porous media as well as cause fluctuations in drying rate. Predicting such phenomenon is therefore essential to creating accurate models of drying. 


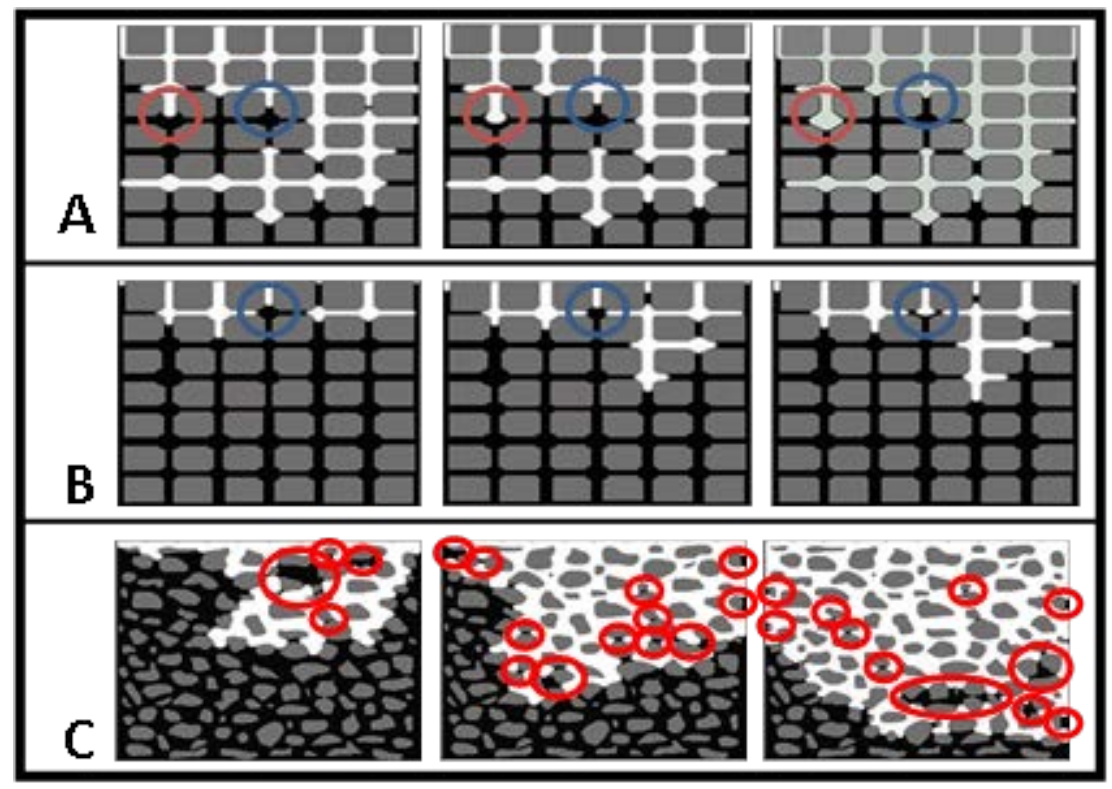

Figure 1: A) Occurance of Haines jump (red) leads ot refilling of vertical throat (blue). B) Effect of surface tension impedes the emptying of pore (blue). C) Large extend of sluster formation.

Apart from the geometry of the porous media, the properties of the wetting fluid also plays a crucial part in the drying. One such property, the surface tension, can prevent the invasion of a pore even though its adjointing throats are empty, as shown in Figure 1B. It can be seen that the pore (circled in blue) does not invade for a considerable amount of time even though two of its adjacent throats are empty. As explained, emptying of pores creates unstable intermediate interfaces. This unstability is due to the high surface energy present at the interface. Therefore, to invade a pore, the surface energy of the interface has to become equal to or larger than the intermediate interfacial energy. This is usually achieved due to reduction in pressure above the interface, and so, increase in surface tension and energy. Hence, pores are not invaded until the surface energy of the pore reaches a threshold value.

The structured porous media shown above is merely an approximation of the actual porous media, which vary widely in shape and size of both pores and throats. Figure $1 \mathrm{C}$ shows a more random type of porous media where the drying phenomena is simulated. Here, the wide range of sizes and shapes lead to clusters formation (red). These clusters may be very small and local to single throats, or large and spanning several pores. These clusters of liquid are bounded by interfaces of high capillarity. This implies that such clusters remain idle until and unless the surrounding pressure reduces sufficiently. It is therefore possible for clusters to exist even after the drying completes. Such bounded liquid can lead to significant problems in drying efficiency and quality of products and so, have to be accounted for. 


\section{Conclusions}

Modelling of drying of porous media has been done in the past using various techniques such as Pore Network Models and Continuum models and have met with some success. Here, we used a Carnahan Starling equation of state based high density ration Lattice Boltzmann Method to model the same under realistic operating conditions. The following key observations were made from this study:

1. Haines jumps cause significant changes in liquid orientation and can lead to unexpected variations in drying rate.

2. Surface energy of the interface plays an important role in deciding which pore to invade and when.

3. Highly randomized and realistic porous media exhibit high levels of liquid entrapment and cluster formation

It can be concluded that the Lattice Boltzmann Method provides an accurate and plausible alternative to continuum and pore network models when it comes to modeling in porous media.

\section{References}

[1] Surasani, V.K., T. Metzger, and E. Tsotsas, Influence of heating mode on drying behavior of capillary porous media: Pore scale modeling. Chemical Engineering Science, 2008. 63(21): p. 5218-5228.

[2] Sánchez-Palencia, E., Non-homogeneous media and vibration theory. Lecture notes in physics. 1980: Springer-Verlag.

[3] Benzi, R., S. Succi, and M. Vergassola, The lattice Boltzmann equation: theory and applications. Physics Reports, 1992. 222(3): p. 145-197.

[4] Quintard, M. and S. Whitaker, Transport in ordered and disordered porous media: volume-averaged equations, closure problems, and comparison with experiment. Chemical Engineering Science, 1993. 48(14): p. 2537-2564.

[5] Nourgaliev, R.R., et al., The lattice Boltzmann equation method: theoretical interpretation, numerics and implications. International Journal of Multiphase Flow, 2003. 29(1): p. 117-169.

[6] El Abrach, H., H. Dhahri, and A. Mhimid, Lattice Boltzmann method for modeling heat and mass transfers during drying of deformable porous medium. AIP Conference Proceedings, 2011. 1453(1): p. 211-216.

[7] Sukop, M.C. and D. Or, Lattice Boltzmann method for modeling liquid-vapor interface configurations in porous media. Water Resources Research, 2004. 40(1): p. W015091W0150911.

[8] Armstrong, R.T. and S. Berg, Interfacial velocities and capillary pressure gradients during Haines jumps. Physical Review E - Statistical, Nonlinear, and Soft Matter Physics, 2013. 88(4).

[9] Bhatnagar, P.L., E.P. Gross, and M. Krook, A model for collision processes in gases. I. Small amplitude processes in charged and neutral one-component systems. Physical Review, 1954. 94(3): p. 511-525. 
[10] Martys, N.S. and H. Chen, Simulation of multicomponent fluids in complex threedimensional geometries by the lattice Boltzmann method. Physical Review E Statistical Physics, Plasmas, Fluids, and Related Interdisciplinary Topics, 1996. 53(1): p. 743-750.

[11] Shan, X. and H. Chen, Lattice Boltzmann model for simulating flows with multiple phases and components. Physical Review E, 1993. 47(3): p. 1815-1819.

[12] Yuan, P. and L. Schaefer, Equations of state in a lattice Boltzmann model. Physics of Fluids, 2006. 18(4).

[13] Kupershtokh, A.L., et al., Stochastic models of partial discharge activity in solid and liquid dielectrics. IET Science, Measurement \& Technology, 2007. 1(6): p. 303-311. 